\title{
In zehn Jahren Nullemissionen? Widersprüche im Paris-Abkommen und ihre Auflösung. Zugleich zu Vorsorgeprinzip und überschätzten Klimaszenarien.
}

\author{
Felix Ekardt, Anika Zorn und Jutta Wieding*
}

\section{Zusammenfassung}

Das Pariser Klima-Abkommen vom Dezember 2015 erfährt öffentlich viel Kritik. Dabei wird seine äußerst ambitionierte Zielsetzung übersehen, die die globale Erwärmung verbindlich auf 1,5-1,8 Grad gegenüber vorindustriellem Niveau begrenzt. Der Beitrag zeigt in der Schnittmenge offener naturwissenschaftlicher Prognosefragen mit dem rechtlichen Vorsorgeprinzip auf, dass damit ein Weg zu globalen Nullemissionen innerhalb kürzerer Zeit als meist angenommen rechtsverbindlich vorgeschrieben ist. Ferner wird deutlich, dass die Politik sogar auf eine Einhaltung der 1,5-Grad-Grenze ausgerichtet werden muss - und dass rechtlich gesehen bei Existenzfragen wie dem Klimawandel nur eine Politik, die mit sehr hoher Wahrscheinlichkeit die Temperaturgrenze einhält, zulässig ist. Das stellt auch die vermeintlichen Klimavorreiter EU und Deutschland vor große Herausforderungen. Nach dem Gesagten müssen Deutschland und die EU im Rahmen der regelmäßigen Anpassung der eigenen Reduktionszusagen gemäß dem Paris-Abkommen ihre Verpflichtungen rasch und drastisch nachschärfen.

Schlagwörter: Paris-Abkommen, Klimawandel, Klimaschutz, Menschenrechte, Vorsorgeprinzip, Klimaszenarien

\section{Zero emissions in 10 years? Contradictions in Paris Agreement and their solution. On precautionary principle and overrated climate scenarios.}

\section{Abstract}

The Paris Agreement of December 2015 is subject to much criticism. This however neglects its very ambitious objective which limits global warming legally binding to 1.5 to 1.8 degrees in comparison to pre-industrial levels. This article shows, based on the overlap of unanswered questions for prognoses in natural science and the legal precautionary principle, that this objective indicates a legal imperative towards zero emissions globally within a short timeframe. Furthermore, it becomes apparent, that policies need to be focussed on achieving the 1.5-degreetemperature limit. And from a legal standpoint with regard to existential matters only those policies are justified that are fit to contribute to reaching the temperature limit with high certainty. This creates a big challenge even for the alleged forerunners of climate policies Germany and EU. Because, according to the objective, EU and Germany have to raise the level of ambition in their climate policies rapidly and drastically.

Keywords: Paris Agreement, climate change, climate protection, human rights, precautionary principle, climate scenarios

${ }^{*}$ Prof. Dr. Felix Ekardt, LL.M., M.A. (felix.ekardt@uni-rostock.de) leitet die Forschungsstelle Nachhaltigkeit und Klimapolitik (FNK) in Leipzig und Berlin (www.nachhaltigkeit-gerechtigkeit-klima.de) und lehrt Öffentliches Recht und Rechtsphilosophie an der Universität Rostock, wo jeweils Jutta Wieding, M.A. mit einem Stipendium der Heinrich-Böll-Stiftung zum weiteren Themenfeld dieses Beitrags promoviert und Anika Zorn, B.Sc. in verschiedenen Projekten tätig ist. Dieser Text entstand im Rahmen einer vom Solarenergie-Förderverein Deutschland e.V. finanzierten Studie und des vom BMBF finanzierten Projekts BIOACID zur Ozeanversauerung. 


\section{Problemstellung und Grundlagen des}

Paris-Abkommens und seiner rechtlichen Verbindlichkeit

Die Erde steht, so lautet eine gut fundierte, von einem breiten naturwissenschaftlichen Konsens getragene Einschätzung (IPCC 2014a; IPCC 2014b; im Überblick Ekardt 2016a: $\$ 1$ B.), vor einer einschneidenden globalen Erwärmung um 3 bis 6 Grad Celsius gegenüber vorindustriellem Niveau im Laufe des 21. Jahrhunderts, die durch (primär) menschlich verursachte hohe Treibhausgasausstöße ausgelöst wird, im Kern - neben Landnutzungsaspekten - durch eine starke Nutzung fossiler Brennstoffe in Bereichen wie Energieerzeugung, Produktion, Landwirtschaft, Gebäudewärme, Stromversorgung und Mobilität. Allein um die Stromversorgung geht es also keinesfalls, auch wenn sich die Debatte in Deutschland darauf mitunter stark konzentriert. Ein Klimawandel in besagter Größenordnung droht nach zitiertem naturwissenschaftlich-ökonomischem Kenntnisstand massive ökonomische Schäden, große Migrationsbewegungen, existenzielle Gefährdungen für Millionen Menschen und in letzter Instanz gewaltsame Auseinandersetzungen um schwindende Ressourcen wie Nahrung und Wasser auszulösen. Noch relativ wenig wird öffentlich wahrgenommen, dass es mit der Reduktion von Treibhausgasemissionen (und fossilen Brennstoffen) zugleich um die entscheidende Gegenmaßnahme gegen die Ozeanversauerung als weiteres Umweltproblem geht. Jene Emissionen sind damit auch ursächlich für die oft flagrante Gefährdung mariner Ökosysteme.

Im Dezember 2015 haben sich die Staaten weltweit auf ein neues globales Klimaschutzabkommen geeinigt. Allseits wird das Paris-Abkommen (Paris Agreement/ nachstehend meist PA) enthusiastisch begrüßt, besonders weil schon das Zustandekommen irgendeiner Vereinbarung im Vorfeld deutlich bezweifelt worden war; gleichzeitig wird seine Wirksamkeit bezweifelt. Generell wird allen Staaten weltweit aufgegeben, die Bemühungen um den Klimaschutz zu intensivieren und auch Maßnahmen der Anpassung an einen teilweise nicht

1 Ein weiterer Faktor für die Ozeanversauerung in Gestalt von Schadstoffemissionen geht zentral auf den Einsatz fossiler Brennstoffe in Industrie, Mobilität und Landwirtschaft zurück. Damit handelt es sich bei Ozeanversauerung und Klimawandel um eng verschnittene Probleme - wobei letzterer die Degradation mariner Ökosysteme weiter vorantreibt. Näheres zur Ozeanversauerung und ihrer Governance: Ekardt/ Zorn 2018. mehr zu verhindernden Klimawandel (Adaptation) und finanzielle Hilfen für vom Klimawandel geschädigte Staaten (Loss and Damage) verstärkt in den Blick zu nehmen. Vielfältig diskutiert wird seitdem, dass die Detailregelungen des Abkommens vage und die konkreten Emissionsreduktionszusagen der Staaten in der Höhe freiwillig und durch eine Vielzahl offener Berechnungs- und Verfahrensfragen durchlöchert sind (näher m.w.N.: Ekardt/Wieding 2016).

Doch vorliegend soll weniger dieser allseits bearbeitete Punkt weiter betrachtet als vielmehr gefragt werden, worin das übergreifende Ziel selbst besteht. Es geht um die Analyse des rechtlich verbindlichen Ziels einer globalen Erwärmungsbegrenzung auf „deutlich unter 2 Grad Celsius über dem vorindustriellen Niveau“ verbunden mit "Anstrengungen ..., um den Temperaturanstieg auf 1,5 Grad Celsius über dem vorindustriellen Niveau zu begrenzen“ (Art. 2 PA). Gleichzeitig heißt es in Art. 4 Abs. 1 PA: „Zum Erreichen des in Artikel 2 genannten langfristigen Temperaturziels sind die Vertragsparteien bestrebt, so bald wie möglich den weltweiten Scheitelpunkt der Emissionen von Treibhausgasen zu erreichen ..., um in der zweiten Hälfte dieses Jahrhunderts ein Gleichgewicht zwischen den anthropogenen Emissionen von Treibhausgasen aus Quellen und dem Abbau solcher Gase durch Senken" herzustellen. Betrachtet man diese beiden unstreitig rechtsverbindlichen Zielnormen von einem physikalischen respektive meteorologischen Standpunkt aus, ergeben sich potenziell Widersprüche, weil Art. 2 PA eine viel frühere Dekarbonisierung als Art. 4 PA verlangen könnte.

Eine rechtsinterpretative Analyse (näher zu dieser weltweit praktizierten juristischen Methode: Ekardt 2016a: $\$ 1$ D. III. 3. ${ }^{2}$ ) von Art. 2 und 4 PA bei gleichzeitiger Rezeption des naturwissenschaftlichen Erkenntnisstandes auf dem Wege einer Literaturanalyse dazu, was solche Ziele denn konkret an Emissionsreduktionen erfordern, ermöglicht deshalb erst eine Aussage, wozu sich die Staaten in Paris eigentlich verpflichtet haben - und wie etwaige dabei entstandene Widersprüche aufgelöst werden können und müssen. Eine Untersu-

2 Rechtsnormen werden grammatisch, systematisch, teleologisch und historisch interpretiert, also nach ihrem Wortsinn, nach ihrem Verhältnis zu anderen Rechtsnormen, nach ihrem Zweck und nach ihrer Entstehungsgeschichte. In aller Regel kommen primär die grammatische und systematische Auslegung zur Anwendung, weil die beiden anderen Zugänge mit verschiedenen Problemen verbunden sind; näher m.w.N.: Ekardt 2016a: $₫ 1$ D. III. 3. 
chung dazu verspricht also deutlicher als bislang $\mathrm{zu}$ zeigen, wo sich die Staaten der Welt künftig mit ihren Emissionsreduktionen hinbewegen müssen. Gemäß Art. 3, 4 PA müssen die in regelmäßigen Abständen zu überprüfenden staatlichen Zusagen stets der Umsetzung des Langfristziels dienen, und entsprechen sie ihm nicht, ist rechtlich schrittweise ihre Anpassung an das Langfristziel geboten. All dem widmet sich deshalb der vorliegende Beitrag. In diesem Kontext stellen sich auch Fragen danach, was uns Klimaszenarien sagen können, wie mit Unsicherheiten umzugehen ist und was unter dem vergleichsmaßstäblichen „vorindustriellen Niveau“ "zu verstehen ist, das in Art. 2 PA zum Rechtsbegriff wird. ${ }^{3}$

\section{Widerspruch zwischen Art. 2 und Art. 4 PA - Nullemissionen global in wenigen Jahren statt Ende des 21. Jahrhunderts?}

Ob die Erreichung eines Gleichgewichts von Emissionen und Senken bis Ende des 21. Jahrhunderts (Art. 4 Abs. 1 PA) und die globale Temperaturgrenze von deutlich unter 2 Grad und Anstrengungen hin zu 1,5 Grad in einem Widerspruch zueinander stehen, hängt teilweise von empirischen Fragen danach ab, wie viel Zeit die Menschheit noch dafür hat, um ihre Emissionen so zu senken, dass die Temperaturgrenze eingehalten wird (dazu sogleich). Der mögliche Widerspruch beider Normen hängt aber auch von einer Rechtsinterpretationsfrage ab, nämlich davon, welche Temperaturgrenze mit „deutlich unter“ 2 Grad gemeint ist. Deutlich unter (oder: weit unter - im Original „well below“) bedarf einer juristischen Auslegung. Der Wortlaut legt, da es eben „deutlich“ weniger als 2 Grad, gleichzeitig aber mehr als 1,5 Grad sein muss, etwa 1,7 oder 1,8 Grad als Temperaturgrenze nahe. Dass „Anstrengungen“ in Richtung der 1,5-Grad-Grenze unternommen werden müssen, kann ferner juristisch nicht heißen, dass

3 Auch die Debatte über die UN Sustainable Development Goals 2030 (SDGs; näher: Ekardt 2016a) erfährt durch Analysen zum Paris-Abkommen eine normative Unterfütterung, eine stärkere Konkretisierung und stärkere rechtliche Verbindlichkeit. Denn die SDGs, die außerdem einen Zugang zu moderner Energie für alle, Ernährungssicherheit und Zugang zu Wasser einfordern, weisen umfassende Bezüge zum Klimawandel oder vielmehr zu einem wirksamen Klimaschutz auf, sind gleichzeitig aber als solche nicht rechtsverbindlich, teils nicht sehr bestimmt oder sogar in sich widersprüchlich. Letzteres betrifft etwa das Nebeneinander von Umweltzielen und klassischer Wachstumsausrichtung. dieses Ziel einfach abgeschenkt werden darf. Vielmehr müssen tatsächlich Maßnahmen ergriffen werden, die weitere Reduktionen im Vergleich $\mathrm{zu}$ einer Grenze von 1,7 oder 1,8 Grad versprechen. Wie weitreichend genau diese Anstrengungen sein müssen, wird zwar vom Wortlaut her nicht explizit ausgeführt. Die Entstehungsgeschichte, also der Hergang der Pariser Klimaverhandlungen, legt aber nahe, dass es im Kern darauf ankommen soll, dass real versucht werden soll, die 1,5 Grad zu erreichen, sofern dies nicht unmöglich ist. Wir werden in Abschnitt 4 noch sehen, dass es für diese Deutung der „Anstrengung“ eine ergänzende grundrechtliche Rechtfertigung gibt.

Inhaltlich wird damit in Art. 2 PA eine Aussage getroffen, die sich von der bisher in den Verhandlungen und in der Öffentlichkeit meist diskutierten Zwei-Grad-Grenze unterscheidet. Dies wird bislang noch relativ wenig bemerkt, hat jedoch potenziell drastische Folgen - nämlich kurzfristig nötige drastische Emissionsreduktionen im globalen Maßstab. Deshalb ist der Zeithorizont der „Zweiten Hälfte“ des 21. Jahrhunderts aus Art. 4 Abs. 1 PA möglicherweise nicht ausreichend für die o.g. Temperaturgrenzen. Dem ist jetzt näher nachzugehen. Um die von der jeweiligen Temperaturgrenze implizierten Emissionsreduktionen zu ermitteln, wird im IPCC-Kontext jeweils mit Wahrscheinlichkeiten gerechnet, wobei auch Abschätzungen vorgenommen werden, da es um zukünftige Sachverhalte geht und das Globalklima von einer Vielzahl relevanter, zudem nicht durchgängig genau bekannter Faktoren beeinflusst wird (näher zu daraus resultierenden Problemen siehe Abschnitt 3). Berechnen lässt sich dies im IPCC-Kontext sodann über Emissionsbudgets, Emissionspfade oder ppm- respektive ppb-Konzentrationen der Treibhausgase in der Atmosphäre ${ }^{4}$ (näher: Buhofer 2016).

Bereits das Ziel einer Temperaturbegrenzung auf deutlich unter 2 Grad globale Erwärmung aus Art. 2 Abs. 1 PA verlangt global in rund zwei Jahrzehnten Nullemissionen, wenn man die Daten des Weltklimarates (IPCC) als Zusammenschluss der weltweiten naturwissenschaftlichen Forschung über die dafür noch maximal möglichen globalen Emissionen und gleiche Pro-Kopf-Emissionsrechte weltweit zugrunde

4 Die Abkürzungen ppm bzw. ppb stehen für parts per million/billion und drücken das Verhältnis zwischen der Anzahl von Gasmolekülen zur Gesamtmolekülzahl trockener Luft aus. 391 ppm bedeuten also $391 \mathrm{CO}_{2}$-Moleküle pro Millionen Luftmoleküle. 
legt; für 1,5 Grad müssten Nullemissionen gar schon rund in einem Jahrzehnt erreicht sein. Vorgerechnet wird dies mit den IPCC-Daten z. B. von Höhne u. a. (2016; ähnlich Rahmstorf 2017; Ekardt/Wieding/Henkel 2015; Rogelj u. a. 2016). Aufgrund der Projektionen von (oftmals langjährig in der Atmosphäre verbleibenden) Treibhausgasemissionen und der Erkenntnisse zur Relation von Treibhausgaskonzentration und Globaltemperatur wird eine Berechnung auf Basis der Daten von IPCC 2014 vorgenommen, die in eine prognostizierte wahrscheinliche globale Erwärmung übersetzt wird. Es werden Szenarien zugrunde gelegt, die mit einer Wahrscheinlichkeit von über 66 \% die Zwei-GradGrenze und $50 \%$ eine 1,5-Grad-Erwärmung einhalten, basierend auf einem verbleibenden Budget von rund 250 GtCO2 für die 1,5-Grad-Grenze ab dem Jahr 2014 (Höhne u. a. 2016; IPCC 2014 hatte ab dem früheren Jahr 2012 - das bereits fünf Jahre verstrichen ist - verbleibende $1000 \mathrm{GtCO} 2$ angesetzt für die deutlich höhere Grenze von 2 Grad; siehe auch Peters 2017). Schaut man jenseits des IPCC, finden sich Budgetaussagen mal für ein grobes Spektrum von 1,5 Grad bis 2 Grad und mal direkt für 2 Grad in der Forschung zwischen 150, 590, 1050 und $1240 \mathrm{GtCO}$, wobei sich die Basisjahre unterscheiden und ferner teils nur Kohlendioxid und teils sämtliche Treibhausgase, also Kohlendioxidäquivalente, erfasst sein sollen (zusammenfassend Rahmstorf 2017; Peters 2017; Figueres u.a. 2017; Rogelj u.a. 2017; weitere aktuelle Betrachtungen von Schellnhuber u.a. 2016; Rahmstorf/Levermann 2017; Berger u.a. 2016; Canadell u.a. 2017; Andersson/Broderick 2017; Steininger/Meyer 2017). Verlangt Art. 2 Abs. 1 PA nach dem Gesagten und den zitierten Quellen für 1,5-1,8 Grad als Grenze globale Nullemissionen in rund (abhängig insbesondere auch von den Emissionspfaden und der angenommenen Klimasensitivität) zehn bis zwanzig Jahren, liegt prima facie ein Widerspruch zwischen Art. 2 Abs. 1 PA und Art. 4 Abs. 1 PA vor.

\section{Unschärfen: Bezugsjahr, Wahrscheinlichkeiten, Klimasensitivität, Friktionen von Szenarien und ein menschenrechtlich gestärktes Vorsorgeprinzip}

Die Rede von Wahrscheinlichkeiten und von Budgets, die aufgrund einer Vielzahl von Annahmen zustande kommen und sich dementsprechend durchaus unterscheiden, erzeugt allerdings die Frage, wie weitgehend die Verpflichtung aus Art. 2 Abs. 1 PA denn nun wirklich ist. Klimamodelle, Schätzungen und Bewertungen unterliegen in der Tat immer Unsicherheiten, und wie dargelegt liefern unterschiedliche Modelle, Annahmen und Szenarien auch verschiedene Ergebnisse (IPCC 2014b: 155; Tollefson 2015; Rahmstorf 2017; Peters 2017). Eine Gesamtbetrachtung verschiedener Modelle, wie sie z.B. den IPCC-Berichten zugrunde liegt, versucht genau auf dieses Phänomen $\mathrm{zu}$ reagieren (Schmidt 2007; Rahmstorf 2017). Die Modelle in sich sind so komplex und mit so zahlreichen Annahmen verbunden (die zudem für Außenstehende wenig transparent sind), dass es den Rahmen dieses Beitrags sprengen würde, einen Vergleich aller Einzelheiten zu versuchen. Dennoch könnte man sich vordergründig fragen, ob die klimapolitisch inaktive Politik dann nicht zumindest im Sinne der für sie günstigsten Berechnung interpretieren darf. Denn selbst wenn man annimmt, dass in liberal-demokratischen Verfassungsordnungen die Politik ihren Entscheidungen so sorgfältig wie möglich ermittelte Fakten zugrunde legen muss, liegt bereits ohne nähere Betrachtung nahe, dass politische Spielräume entstehen, wenn die Faktenlage durch Ungewissheiten geprägt ist (Meßerschmidt 2000; Ekardt 2016a: $\$ 5$ C. II. 2.; Calliess 2001). Im Folgenden soll jedoch zweierlei gezeigt werden. Zum einen deuten mehrere Faktoren darauf hin, dass die Berechnungen eher zu großzügig ausfallen, weswegen der Blick primär auf die kleinen und nicht auf die großzügigen Budgets fallen muss, wenn eine realistische Prognose gesucht wird. Zum anderen darf, juristisch betrachtet, die Politik beim Klimawandel wegen dessen existenzieller Bedeutung für die Menschheit kein nennenswertes Risiko eingehen.

Zutreffend ist zunächst, dass bei der Berechnung künftiger Klimaentwicklungen Faktoren involviert sind, die zu unscharfen Aussagen führen. Generell sind Aussagen über die Zukunft nie völlig gewiss, was besonders angesichts der Komplexität von Nachhaltigkeitsfragen regelmäßig betont wird (Ekardt 2016a: $\$ 5$ C. II. 1.). Welches Budget errechnet wird, hängt folgerichtig von einer Reihe von Annahmen ab. Ein Faktor von vielen für die Unsicherheit in Bezug auf die genaue Klimawirkung von Emissionen entsteht durch die Speicherkapazitäten für $\mathrm{CO}_{2}$ in den Ozeanen, die Sammlung von kurzlebigen Aerosolen in der Atmosphäre, die zu einer verzögerten Wirkung anthropogener Treibhausgasemissionen führen. Insbesondere Ozeane spielen eine zentrale Rolle, da sie sich nur langsam und in mehreren Schichten erwärmen. Je kälter sie sind, desto höher die Speicherkapazität für Treibhausgasemissionen. Positiver Nebeneffekt ist, dass z.B. Methan mit sehr starker Klimawirkung im Laufe von zehn Jahren aus der Atmo- 
sphäre verschwindet. Bei einem plötzlichen Stopp von $\mathrm{CO} 2$-Emissionen wiederum würden auch die Aerosole - Schadstoffpartikel - aus der Luft verschwinden und so den Albedo-Effekt aufheben, sodass es auch dadurch zumindest kurzfristig zu einer Beschleunigung der Erwärmung kommt (näher: Mauritsen/Pincus 2017). Bruttoinlandsprodukt und Bevölkerungsentwicklung wirken als zusätzliche Ursache für Unschärfen in den Prognosen (Drouet/Emmerling 2016; breit $\mathrm{zu}$ regionalen Szenarien auch Rose u.a. 2017). Wesentlich für die Budget-Berechnung ist ferner, ob man von einer sofortigen Absenkung der Emissionen ausgeht oder annimmt, dass die Emissionen noch einige Jahre steigen. Zudem ist das Thema Klimaschutz noch mit anderen gesellschaftlichen Großthemen wie Wachstum, internationalem Handel, Digitalisierung, Automatisierung, Globalisierung u.a.m. verflochten, deren genaue weitere Entwicklung ebenfalls von vielfältigen und komplexen Zusammenhängen beeinflusst wird. All das ist nicht grundlegend durch die den Budgetberechnungen zugrunde liegenden Modelle und Szenarien zu beheben. Szenarien sind weder eine sichere Prognose, noch erschöpfen sie auch nur den Möglichkeitsraum künftiger Entwicklungen, und erst recht sind sie nicht normativ (weitere Probleme wie die oft nicht offen gelegten Hintergrundannahmen werden näher betrachtet bei Ekardt 2017; exemplarisch trotz umfassender und gründlicher Arbeiten Bodirsky u.a. 2015 und Wiebe u.a. $2015^{5}$ ). Da man bezogen auf die diversen unwägbaren Ereignisse auch deren Eintrittswahrscheinlichkeit in der Regel nicht genau kennt, können Budgets ohnehin nur eine Abschätzung, aber nicht im strengen Sinne eine Berechnung sein, denn mit ungewissen Wahrscheinlichkeiten kann man mathematisch nicht rechnen, selbst bei größter Expertise nicht.

Auch wenn man also die Klimazukunft nicht exakt kennt, sprechen dennoch wie angedeutet einige starke Anhaltspunkte dafür, dass innerhalb der verschiedenen Abschätzungen eher die kleinen Restbudgets richtig liegen.

Erstens beziehen sich viele Berechnungen auf 2 Grad, was mehr ist als die gemäß Art. 2 Abs. 1 PA

5 Raftery 2017 u.a. gehen davon aus, dass ihre Zahlen aufgrund von Vergleichsrechnungen aus der Vergangenheit validiert werden können: Das Modell angewandt auf eine Hochrechnung beginnend von 1950 und 1980 für jeweils die nächsten 30 Jahre ergab selbst bei einer großen Emissionssteigerung in China zwischen 2000 und 2010 eine 90-prozentige Übereinstimmung mit der realen Entwicklung. Ob dies allerdings über den wesentlich längeren Zeitraum bis Ende des Jahrhunderts ebenfalls zutreffend ist, ist fraglich. zulässigen „deutlich unter 2 Grad“. Rechnet man so, landet man nicht nur bei höheren Budgets, sondern man beschönigt auch das Ausmaß der Herausforderung - Nullemissionen in kurzer Zeit. Daran geht beispielsweise auch der langjährige Diskurs über die Vereinbarkeit von Wachstum und Umweltschutz vorbei, der eben nicht dieses Ambitionsniveau zugrunde legt (hierzu Ekardt 2016a: \$1 B. III.; Hoffmann 2015: 12ff.; Jackson 2011: 81ff.; Piketty 2015: 29ff.; Moreno/Speich Chassé/ Fuhr 2015: 28; wenig kritisch Paqué 2010: 96ff.).

Zweitens ist die Einbeziehung der Nicht-Kohlendioxid-Emissionen nicht in allen Budgets gegeben. Andere Treibhausgase halten sich zwar anders als Kohlendioxid nicht lange in der Atmosphäre; dennoch spielen auch sie für den Klimawandel eine entscheidende Rolle (Buhofer 2017). Schließt man aus einem reinen Kohlendioxid-Budget auf den Handlungsbedarf bei Treibhausgasen, verringert man das Ausmaß der Herausforderung künstlich.

Drittens werden Budgetberechnungen auch dadurch relativ großzügig, dass das Referenzjahr des „Vorindustriellen Niveaus“, das gemäß Art. 2 Abs. 1 PA gilt, spät datiert wird und dadurch die globale Erwärmung, die bereits erfolgte, geringer eingeschätzt wird. ${ }^{6}$ Generell ist ein Referenzpunkt nötig, um überhaupt einheitliche Berechnungen durchführen zu können. Gängig ist primär ein Basisjahr von 1860 bis 1880 für die Berechnung der Temperaturgrenze, doch wird auch 1750 genannt (Peters 2017; Rahmstorf 2017). Damit schließt sich die Frage an, wann genau Industrialisierung respektive eine Zunahme der Emissionen eigentlich begann. Der IPCC zieht die Grenze zunächst im Jahr 1750 (IPCC 2013: 1456). Berechnungen und Schätzungen bezüglich der Wahrscheinlichkeit der globalen Durchschnittserwärmung werden indes mit Bezug auf das Jahr 1850 bzw. 1870 gemacht, da für die Zeit vor dem 19. Jahrhundert nur sehr wenige Daten von Temperaturmessungen vorliegen und sich diese vorwiegend auf die Nordhalbkugel beschränken (IPCC 2013: 953-1028, 1029-1136; IPCC 2014a: 64). Durch den CO2-Anstieg vor 1850 ist eine Temperatursteigerung von 0,1 bis 0,2 Grad Celsius zu verzeichnen (Schurer u.a. 2017). Letztlich ist die Frage, was „vorindustrielles Niveau“ meint, jedoch nicht nur eine freischwebende empirische Dis-

6 Einen Überblick über verschiedene Arten von Szenarios bietet auch die Seite https://www.iea.org/publications/ scenariosandprojections/. Die IPCC-Daten werden auch zusammengestellt auf der Seite https://www.carbonbrief.org/ analysis-only-five-years-left-before-one-point-five-c-budgetis-blown. 
kussion darüber, was zu den jeweiligen Zeiten denn für ein Emissionsniveau herrschte. Wenn Art. 2 Abs. 1 PA als Rechtsdokument den Alltagsbegriff „,vorindustriell“ verwendet, erscheint ein Ansetzen bei 1750 geboten. Denn konkret damals begann die industrielle Revolution in den westlichen Staaten - und nicht etwa erst zwischen 1860 und 1880 .

Viertens können bisherige Berechnungen auch deshalb als eher zu großzügig erscheinen, wenn man andere Annahmen zur Klimasensitivität trifft. GleichgewichtsKlimasensitivität (equilibrium climate sensitivity/ECS; näher: Buhofer 2016) beschreibt den Temperaturanstieg bei einer Verdopplung der $\mathrm{CO}_{2}$-Äquivalente in der Atmosphäre und ist damit entscheidend für Klimamodelle und schlussendlich auch für die in Art. 2 Abs. 1 PA geforderte Erwärmungsbegrenzung (IPCC 2013: 1451). Laut IPCC (2013: 16) liegt die ECS wahrscheinlich zwischen 1,5 und 4,5 Grad Celsius. Neuere Studien von Friedrich u.a. (2016) und Storelvmo u.a. (2016) legen nahe, dass die ECS unterschätzt wurde und eher im oberen Bereich dieser Spanne oder gar darüber liegt. So kam bei paläoklimatischen Untersuchungen heraus, dass die Klimasensitivität vom Klimazustand abhängt und in Warmphasen (wie der jetzigen) signifikant höhere Werte annimmt - nach den Berechnungen von Friedrich u.a. (2016) 4,88 Grad Celsius, was die IPCC-Spannweite deutlich überschreitet. Ein weiterer Unsicherheitsfaktor bei der Bestimmung der ECS ist die Bewölkung, da diese den Strahlungshaushalt der Erde maßgeblich mitbestimmt. Storelvmo u.a. (2016) kommen bei einer Studie zu dem Ergebnis, dass deutlich mehr Solarstrahlung nicht, wie bisher angenommen, von den Wolken zurück in das All reflektiert wird, sondern die Wolkenschicht durchdringt und damit die Erde stärker erwärmt. Je nach Bewölkung kann eine bis zu 1,3 Kelvin höhere Klimasensitivität auftreten als bisher erwartet (Mauritsen/Pincus 2017).

Fünftens basieren die Budgetberechnungen darauf, eine relativ große Wahrscheinlichkeit der Verfehlung der jeweiligen Temperaturgrenze hinzunehmen. Jedoch ist es bei einer existenziellen Bedrohung wie dem Klimawandel prima facie verblüffend, sich mit Schutzwahrscheinlichkeiten wie 50 oder $66 \%$ zu begnügen (auch wenn hundertprozentige Sicherheit für in der Zukunft liegende Sachverhalte naheliegenderweise nicht $\mathrm{zu}$ erreichen sein wird). Insoweit ist auch $\mathrm{zu}$ bedenken: Einige kritische Klima-Umschlagpunkte wie das Schmelzen des Grönländischen oder Westantarktischen Eisschildes oder für die Korallenbleiche werden wahrscheinlich schon bei einer Erwärmung von deut- lich unter 2 Grad erreicht (Frieler u.a. 2012; Rahmstorf/ Levermann 2017: 3f.). Eine Zielmarge von 1,5 bis 1,8 Grad bewegt sich also keinesfalls in einem ungefährlichen Bereich, den man deshalb auch nur mit begrenzter Wahrscheinlichkeit anzusteuern bräuchte.

Im Anschluss an die Darlegungen, dass empirischprognostisch ein geringes Budget im genannten Spektrum und somit eine Dekarbonisierung innerhalb weniger Jahre als im Lichte des Art. 2 Abs. 1 PA als gemeint erscheint, lassen sich rechtlich weitere Aussagen treffen. Dass die genannten Umstände für das Ansetzen einer möglichst geringen Schädigungswahrscheinlichkeit und ergo für einen möglichst raschen drastischen Klimaschutz in der Interpretation des Art. 2 Abs. 1 PA sprechen, ergibt sich juristisch aus zwei Gesichtspunkten.

Zum einen gilt, dass die Politik sich nicht mit 1,7 bis 1,8 Grad zufrieden geben darf, sondern versuchen muss, die 1,5 Grad zu halten. Denn wie gesagt: Dass „Anstrengungen“ in Richtung der 1,5-Grad-Grenze unternommen werden müssen, kann juristisch nicht heißen, dass dieses Ziel einfach abgeschenkt werden darf. Vielmehr müssen tatsächlich Maßnahmen ergriffen werden, die weitere Reduktionen im Vergleich zu einer Grenze von 1,7 oder 1,8 Grad versprechen.

Zum anderen weisen menschenrechtliche Verpflichtungen zum Klimaschutz, zum Schutz der elementaren Freiheitsvoraussetzungen Leben, Gesundheit und Existenzminimum, in diese Richtung (ausführlich dazu Ekardt 2016a: $\$ \$ 4$, 5; Rajamani 2010; Knox 2013; Skillington 2012; Verheyen 2005; allgemein zum Umweltschutz auch Unnerstall 1999; Schmidt-Radefeldt 2000; Calliess 2001; Koch 2000). Diese Verpflichtung wird zugleich explizit in der Präambel des Paris-Abkommens in Erinnerung gerufen - wissend darum, dass eine globale Erwärmung, die die Nahrungs- und Wasserversorgung beeinträchtigen und damit (neben Naturkatastrophen) Migrationsbewegungen und Kriege um schwindende Ressourcen wahrscheinlicher machen kann, die Grundlagen der menschlichen Zivilisation in Gefahr bringen kann. Zwar unterliegen menschliche Verpflichtungen in puncto Klimaschutz prima facie Spielräumen der Staaten (wegen der gegenläufigen Freiheitsrechte etwa von Unternehmen und Konsumierenden), die lediglich durch einzuhaltende Abwägungsregeln eingegrenzt werden. Eine Abwägungsregel lautet jedoch, dass der politische Entscheidungsspielraum dort endet, wo ein politisches Tun oder Unterlassen das freiheitlich-demokratische System als solches zu gefährden beginnt (näher: Ekardt 2016a: $\$ 5$ C. I.; zu weiteren Regeln ferner Calliess 2001 und Susnjar 2010). Just dies droht ein im eben geschil- 
derten Sinne ungebremster Klimawandel zu tun. Damit ist ein strenger Klimaschutz menschenrechtlich geboten.

Dies wirft natürlich wiederum die Frage auf, wie stark und wie schnell dafür die Emissionen reduziert werden müssen. Und es liegt auch auf der Hand, dass die geschilderten Entwicklungen denkbar, aber keineswegs sicher sind. Jedoch schützen die Grundrechte auch vor möglichen und nicht nur vor sicheren Gefährdungen, sofern die Gefährdung im Eintrittszeitpunkt sonst irreversibel wäre (und genau so wäre es für den Klimawandel). Denn sonst liefe der Grundrechtsschutz leer (Koch 2000; Ekardt 2016a: $\$ 5$ C. II. 2.). Die Menschenrechte enthalten also auch ein Vorsorgeprinzip; selbst wenn man dies bestreiten würde, wäre jedenfalls unstreitig, dass das Vorsorgeprinzip (auch) unabhängig von den Menschenrechten im nationalen, EU- und Völkerrecht existiert, sichtbar z.B. in der Klimarahmenkonvention in Art. 3 Abs. 3 KRK, im Vertrag über die Arbeitsweise der EU in Art. 191 AEUV oder im deutschen Grundgesetz in Art. 2oa GG. Vorsorge meint Vorkehrungen angesichts von langfristigen, kumulativen oder ungewissen Schadensverläufen (Arndt 2009; Maurmann 2008; Monien 2014; konkret zu seiner Reichweite Ekardt 2016a: $\$ 5$ C. II. 2.). Genau darum geht es beim Klimawandel. Diesbezüglich macht der menschenrechtliche Bezug nur noch deutlicher, was dem Vorsorgeprinzip auch sonst inhärent ist: Je größer das drohende Schadensausmaß im Eintrittsfall sein würde, desto weitreichender ist der gebotene Schutz, auch zu Lasten der genannten konkurrierenden Güter wie der wirtschaftlichen Freiheit. Bei existenziellen Gefahren genügen deshalb keine moderaten Wahrscheinlichkeiten für deren Abwehr, auch wenn hundertprozentige Sicherheit bezogen auf zukünftige Vorgänge naturgemäß nicht erreichbar ist. Es ist in der Budgetdebatte damit rechtlich geboten, von den eher pessimistischen Zahlen auszugehen und dementsprechend weitgehende Anstrengungen (weltweit) zur kurzfristigen Dekarbonisierung zu ergreifen. ${ }^{7}$ Folglich sind selbst die im letzten Abschnitt im Querschnitt

$7 \quad$ Ferner kann man mithilfe der aus den menschenrechtlichen Freiheits- und Freiheitsvoraussetzungsgarantien ableitbaren Abwägungsregeln (Geeignetheit, Erforderlichkeit, Leistungsfähigkeit, Verursacherprinzip u.a.m.) neben der gemeinsamen Klimaschutzpflicht sogar eine ungefähre Lastenverteilung zwischen den Staaten ableiten. Da die Länder der EU, beispielsweise Deutschland, bislang pro Kopf besonders hohe Emissionen hatten, die sich oft noch in der Atmosphäre befinden, verstärkt das den Handlungsdruck hierzulande - über das Gesagte hinaus - noch weiter. Näher dazu Ekardt/Wieding/Henkel 2015; Rahmstorf 2017; Ekardt 2016a: $\$ 5$ C. IV. - und ferner kurz im letzten Abschnitt. verschiedener Budgetberechnungen verbleibenden zehn bis zwanzig Jahren für jene Dekarbonisierung eher zu großzügig bemessen (dazu, dass eine solche Aussage wegen der bestehenden Unsicherheiten und aus Gewaltenteilungsgründen nicht exakt quantifiziert werden kann, sondern parlamentarische Spielräume verbleiben, siehe Ekardt 2016a: $\$ 5$ B.-C.; Calliess 2001; Susnjar 2010; Ekardt 2018; zur Frage der Wirtschaftlichkeit eines Dekarbonisierungspfades verglichen mit einem Business-as-usual mit möglichen katastrophalen Folgen Ekardt 2016b).

\section{Rechtliche Auflösung des Verhältnisses zwischen Art. 2 und Art. 4 PA}

Inhaltlich wird mit Art. 2 PA also eine sehr ambitionierte Aussage getroffen. Und es wird eine Aussage getroffen, die sich von der bisher in den Verhandlungen und in der Öffentlichkeit meist diskutierten Zwei-Grad-Grenze unterscheidet. Dies wird bislang noch relativ wenig bemerkt, hat jedoch wie gesehen drastische Folgen - nämlich kurzfristig nötige drastische Emissionsreduktionen im globalen Maßstab. Das in Art. 2 Abs. 1 PA zusätzlich genannte Ziel von Anstrengungen, die Erwärmung auf 1,5 Grad zu begrenzen, verschärft diese ohnehin schon markante Aussage weiter. Wenn es die Vertragsstaaten gemäß Art. 4 Abs. 1 PA „beabsichtigen“, den Höhepunkt der Emissionen „möglichst“ bald zu erreichen und es in der zweiten Hälfte des 21. Jahrhunderts zu schaffen, ihre Emissionen vollständig zu neutralisieren, reicht das für die Erfüllung des Art. 2 Abs. 1 PA also nicht aus. Angesichts des Regelungswiderspruchs zwischen Art. 2 Abs. 1 und 4 Abs. 1 PA ist rechtsinterpretativ die Vorrangigkeit zu ermitteln. Dabei sprechen mehrere Argumente für eine Vorrangigkeit des Art. 2 Abs. 1 PA. Es handelt sich dabei im Wesentlichen um eine systematische Auslegung, also um eine Norminterpretation, die den Zusammenhang verschiedener Normen untereinander bedenkt.

Für die Vorrangigkeit des Art. 2 Abs. 1 PA spricht erstens, dass es sich um die übergreifende Zielnorm des Paris-Abkommens handelt. Art. 4 PA widmet sich, dem nachgeordnet, stattdessen den konkreten Strategien zur Umsetzung dieses Ziels. Dass dieses Umsetzungsverhältnis besteht, wird in Art. 3 und 4 Abs. 1 PA zweimal ausdrücklich angesprochen. Der Orientierungspunkt und damit die höherrangige Norm ist ergo Art. 2 PA.

Zweitens: Normhistorisch und vom Normzweck her ist Art. 4 Abs. 1 PA (auch wenn er vom Wortlaut 
her für alle Staaten gilt, denn dort ist von „Parties“ die Rede) vor allem so gemeint, dass die Entwicklungsund Schwellenländer (nicht aber die Industrieländer) noch Zeit haben sollen für ihre Emissionsreduktionen (das zeigt auch Art. 4 Abs. 4 PA). Auch für die Entwicklungsländer lässt sich dies - siehe oben - allerdings so nicht durchhalten, weil sonst wiederum Art. 2 Abs. 1 PA verletzt wäre. Jedenfalls verdeutlicht der Gesichtspunkt, dass hier primär eine Staatengruppe gemeint ist, allerdings zweierlei: Art. 4 Abs. 1 PA hat eher einen operativen und dienenden Charakter. Und speziell für die Industriestaaten kann man stark bezweifeln, ob für sie der Art. 4 Abs. 1 PA überhaupt eine zu Art. 2 Abs. 1 PA konträre Aussage treffen will.

Ein drittes, systematisches Argument kann man wie folgt formulieren: Würde man das Normverhältnis zugunsten des Art. 4 interpretieren, würde Art. 2 verletzt werden. Interpretiert man dagegen zugunsten des Art. 2, wird Art. 4 nicht verletzt - er wird dann eher überboten, denn Art. 4 PA fundiert kein Verbot, schneller $\mathrm{zu}$ sein als dort vorgegeben. Die Formulierung „deutlich unter zwei Grad gehalten“ in Art. 2 PA unterstreicht auch, dass die Emissionen nicht erst beliebig steigen und sodann wieder auf jenes Temperaturmaß zurückgeführt werden dürfen. Art. 3 PA macht deutlich, dass die Nationalstaaten dem Ziel aus Art. 2 PA eben durch eine sukzessive Steigerung ihres Ambitionsniveaus (zum bisherigen Niveau der Anstrengungen sogleich) gerecht werden müssen. Es heißt dort: „Zur Verwirklichung des in Art. 2 genannten Zieles dieses Übereinkommens sind von allen Vertragsparteien als national festgelegte Beiträge zu der weltweiten Reaktion auf Klimaänderungen ehrgeizige Anstrengungen im Sinne der Artikel 4, 7, 9, 10, 11 und $13 \mathrm{zu}$ unternehmen und zu übermitteln. Die Anstrengungen aller Vertragsparteien werden im Laufe der Zeit eine Steigerung darstellen."

Für das Primat von Art. 2 PA gegenüber Art. 4 PA spricht noch ein vierter, systematischer Umstand. Gemeint ist, dass das Paris-Abkommen rechtssystematisch als Konkretisierung der Klimarahmenkonvention respektive als umsetzender Rechtsakt in deren Rahmen auftritt. Insbesondere Art. 2 KRK verpflichtet die Staaten als übergreifende Zielnorm des gesamten Klimavölkerrechts zur Vermeidung gefährlicher anthropogener Störungen des globalen Klimas. Diese Störung kann nach dem Gesagten jedoch nur vermieden werden, wenn Art. 2 Abs. 1 PA gegenüber Art. 4 Abs. 1 PA interpretativ der Vorrang gegeben wird, weil die Vorgaben des Art. 4 Abs. 1 PA eben eine substanzielle globale Erwärmung zulassen würden. Gemäß Art. 31 Abs. 3 Wiener Vertragsrechts-Konvention (WVRK) ist eine solche systematische Interpretation des ParisAbkommens im Lichte eines anderen völkerrechtlichen Vertrags ausdrücklich ein Teil des Interpretationsaktes. Das Gesagte gilt umso mehr, als menschenrechtliche Garantien wie dargelegt in die gleiche Richtung weisen.

Auf eines ist am Rande noch hinzuweisen. Eine ausdrückliche Aussage $\mathrm{zu}$ einem Ausstieg aus den fossilen Brennstoffen bei Strom, Wärme, Mobilität, Kunststoffen und Mineraldünger zugunsten von erneuerbaren Energien, Energieeffizienz und Suffizienz findet sich in Art. 2 Abs. 1, Art. 4 Abs. 1 PA nicht, obwohl genau hier der zentrale strategische Ansatzpunkt für Klimaschutz liegt. Die dortige Vorgabe, Emissionen zu neutralisieren, könnte statt eines Ausstiegs aus Öl, Gas und Kohle vordergründig als Einladung zu Geo-Engineering-Maßnahmen verstanden werden. Gemeint sind damit insbesondere Eingriffe in die Atmosphäre oder die Ozeane (oder die unterirdische Speicherung von abgeschiedenem $\mathrm{CO}_{2}$ etwa aus Kohlekraftwerken), um die Sonneneinstrahlung zu reduzieren oder mehr Treibhausgase zu binden. Die Diskussion darüber ist zu komplex, um hier en passant aufgenommen zu werden. Doch spätestens wenn sich solche Optionen als ganz oder im Wesentlichen undurchführbar erweisen, bleibt nur der fossile Ausstieg und der Übergang zu $100 \%$ erneuerbaren Energien, eine gesteigerte Energieeffizienz und ggf. auch Suffizienz (und auf im Umfang begrenzte, dafür aber direkt verfügbare Techniken der Kompensation wie Aufforstungen oder Wiedervernässung von Mooren). Und genau davon ist jedenfalls im Zeithorizont des Art. 2 Abs. 1 PA auszugehen. Denn entsprechende Technologien stehen aktuell nicht marktreif zur Verfügung - womit sich auch mögliche Diskussionen über Kosten und Risiken weitgehend erübrigen. Letztere wären nötig, um die selbst bei einem vollständigen fossilen Ausstieg und weiteren Änderungen (wie einer stärker pflanzlichen Ernährung) verbleibenden Restemissionen zu kompensieren (zur Kontroverse um Negativemissionen UNEP 2016; Ekardt 2016a: $\$ 1$ B.; Höhne u.a. 2016: 11 f.; Smith u.a. 2016; Hennig 2017).

Das Gesagte wird auch nicht dadurch hinfällig, dass die Zielerreichung des Art. 2 Abs. 1 PA schlicht unmöglich ist und sich damit die Frage nach dem Verhältnis der Normen vermeintlich auf andere Weise erledigt. Unmögliches ist rechtlich zwar nie geschuldet, wie andernorts ausführlicher gezeigt wurde (Ekardt 2016a: $\$ 3$ G.). Unmöglich ist die Zielerreichung des 
Art. 2 Abs. 1 PA jedoch wahrscheinlich nicht, wie wir sahen: Das wäre sie nur, wenn die Erreichung naturgesetzlich unmöglich wäre, selbst in einem Extremfall wie dem sofortigen Aussterben der Menschheit. Nicht erübrigen lassen sich diese Fragen durch das beliebte Statement, man solle gar nicht über Ziele reden. Denn Art. 2 Abs. 1 PA gilt rechtsverbindlich (kritisch zu Temperaturgrenzen Knutti u.a. 2016). Ebenfalls fehlgehen würde es, wenn man darauf verweist, dass Zielbestimmungen im Recht nicht immer verbindlich sind; denn Art. 2 Abs. 1 PA ist, anders als sonstige Ziele wie allgemein der Klimaschutz, präzise und unmissverständlich formuliert, und außerdem machen Art. 3 und 4 Abs. 1 PA deutlich, dass Art. 2 Abs. 1 PA die verbindliche Grundlage für alle Klimaschutzmaßnahmen ist. Erst recht kein Gegenargument wäre es, darauf zu verweisen, dass die meisten Staaten sich den Art. 2 Abs. 1 PA vielleicht als bloße unverbindliche Lyrik vorgestellt haben, als sie ihm zustimmten; denn im Recht gilt der Wortlaut von Normen und nicht das, was sie bei einer entstehungsgeschichtlichen Motivforschung ergeben.

Mancher mag abschließend fragen: Wird das Paris-Abkommen durch den angekündigten USAustritt nicht ohnehin hinfällig? Art. 28 PA sagt dazu: „(1) Eine Vertragspartei kann jederzeit nach Ablauf von drei Jahren nach dem Zeitpunkt, zu dem dieses Übereinkommen für sie in Kraft getreten ist, durch eine an den Verwahrer gerichtete schriftliche Notifikation von diesem Übereinkommen zurücktreten. (2) Der Rücktritt wird nach Ablauf eines Jahres nach dem Eingang der Rücktrittsnotifikation beim Verwahrer oder zu einem gegebenenfalls in der Rücktrittsnotifikation genannten späteren Zeitpunkt wirksam." Wobei der Austritt den Gesamtbestand des Abkommens nicht tangiert, weil kein Außerkrafttreten vorgesehen ist, wenn das Inkrafttreten einmal erfolgt ist. Wenn man dagegen die Ratifikation durch die USA als solche bestreitet, wäre ein Austritt sofort möglich. Dies ist allerdings bislang nicht geschehen. Ob dieses Bestreiten, wenn die Ratifikation (wie im Oktober 2016 geschehen) schon hinterlegt wurde, völkerrechtlich überhaupt einen Austritt erreichen kann und zugleich womöglich das ganze Abkommen wegen nunmehr geringerer erfasster globaler Emissionsmengen als nicht wirksam in Kraft getreten erscheinen lässt, ist zudem sehr zweifelhaft. Ungeachtet dieser rechtlichen Betrachtung ist es für die Wirksamkeit des Paris-Abkommens ersichtlich ungünstig, wenn Staaten mit hohen Emissionen sich aus dem internationalen Klimaschutz zurückzuziehen versuchen.

\section{Pariser Langfristziel, EU-Klimapolitik und die Wachstumsdebatte}

Ungeachtet des somit einschneidenden und rechtsverbindlichen Ziels einer globalen Erwärmungsbegrenzung auf 1,5 bis 1,8 Grad sind die nationalen Beiträge bisher nicht ausreichend, die Angaben des Abkommens zu finanziellen Unterstützungen vage und mögliche Sanktionsmechanismen schlicht nicht vorhanden (näher dazu und zum Folgenden m.w.N.: Ekardt/ Wieding 2016; Ekardt 2016; ferner Hennig 2017; avant la lettre Becker/Richter 2015). Im Wesentlichen sämtliche Staaten werden mit ihren bisherigen Klimazielen und erst recht ihren Instrumenten jene ambitionierten Temperaturgrenzen weit verfehlen. Der Emissions Gap Report des UN-Umweltprogramms evaluiert, ob die Summe der bisher eingereichten NDCs ausreicht, um die für die Ziele des Paris-Abkommens notwendige Trendwende im Klimaschutz herbeizuführen. Dabei wurde als Grundlage einerseits die 2-Grad-Marke (noch nicht angepasst an Art. 2 PA: "deutlich unter“ $2 \mathrm{Grad}$ ) und andererseits die angestrebten 1,5 GradBegrenzung genommen und die bisherigen Anstrengungen als unzureichend aufgewiesen (UNEP 2016; UNEP 2017; ferner z.B. Höhne u.a. 2016; Rogelj u.a. 2016; Ekardt/Wieding/Henkel 2015; siehe auch Figueres u.a. 2017).

Wenn man zwischen den Staaten ferner die meistens diskutierten menschenrechtlich-abwägenden Verteilungsmaßstäbe (primär Leistungsfähigkeit und historische Verantwortung im Sinne bisher erfolgter Emissionen seit 1990, siehe dazu auch Art. 4 Abs. 4 und 9 Abs. 3 PA) annimmt, müssen die EU-Länder wie etwa Deutschland eigentlich mehr Emissionen reduzieren, als sie aktuell überhaupt ausstoßen. Dies klang bereits kurz an und würde dann zweistellige jährliche Milliarden-Zahlungspflichten zur Unterstützung der Emissionsreduktion im globalen Süden bedeuten, möglicherweise noch begleitet von ebenfalls hohen Zahlungen für Adaptation und Klimawandelfolgeschäden; dabei ergeben sich bereits für eine Zwei-Grad-Grenze plus eine eher geringe Erreichungswahrscheinlichkeit einer 1,5-Grad-Grenze und ein Zieljahr 2050 in einer Berechnung minus 162 \% Emissionsreduktionsverpflichtungen etwa für Deutschland (unter Verwendung der Daten aus IPCC 2014 und Schellnhuber 2015 im Einzelnen vorgerechnet bei Ekardt/Wieding/Henkel 2015: 6ff.). Dies ergibt dann übersetzt z.B. Nullemissionen plus zweistellige jährliche Milliarden-Zahlungspflichten allein schon für die Mitigation im globalen 
Süden. Geht man nach dem Paris-Abkommen nunmehr von „deutlich unter 2 Grad“ oder sogar 1,5 Grad aus, verschärft das die Zahlen weiter. ${ }^{8}$ Die Zielverfehlung bezogen auf Art. 2 Abs. 1 PA durch Staaten wie Deutschland und den Staatenbund EU (aber letztlich auch durch praktisch alle anderen Staaten) ist bereits in Art. 3, 4 PA angelegt, weil es gemessen an der sehr ambitionierten verbindlichen Zielsetzung des Art. 2 Abs. 1 PA etwas kontradiktorisch wirkt, den Staaten zunächst nur freiwillige nationale Emissionsreduktionen als Umsetzung der allgemeinen Zielstellung abzuverlangen, die allerdings später nachgebessert werden müssen. Nach dem Gesagten müssen Deutschland und die EU ergo ihre Verpflichtungen rasch und drastisch nachschärfen (andere Staaten allerdings auch).

Schon für die Vergangenheit ist die Rede vom Klimavorreiter EU bzw. Deutschland keineswegs so berechtigt, wie allgemein angenommen wird (kritisch Becker/Richter, Momentum Quarterly 2015: 3ff.; Hennig 2017; Ekardt 2016a: \$ 1 B. III.; Moreno/ Speich Chassé/Fuhr, Carbon Metrics, 2015: 13ff.; Ekardt 2016b). Das manifestiert sich bereits darin, dass auch diese Vertragsparteien in Paris nicht viel $\mathrm{zu}$ einem besseren Verhandlungsergebnis, als es soeben geschildert wurde, beigetragen haben. Die EU hat in Paris als (supra-)nationalen Klimaschutzbeitrag im Sinne von Art. 4 Abs. 2 PA Treibhausgasemissionen von minus 40 $\%$ bis 2030 versprochen. Hinter der von der EU gleichzeitig mitgeforderten o.g. Temperaturgrenze bleibt dies weit zurück. Hätten westliche Industriestaaten indes deutlich stärkere Reduktionsziele und eine stärkere finanzielle Unterstützung der Entwicklungsländer im Rahmen von Mitigation, Adaptation sowie Loss and Damage angeboten, wären auch klarere Klimaschutzverpflichtungen für die Staaten weltweit denkbar gewesen. Hintergrund ist, dass die EU und Deutschland trotz der selbst zugeschriebenen positiven Rolle unverändert pro Kopf bei um ein Vielfaches überhöhten Emissionen stehen, wenn man die genannten Temperaturgrenzen in den Blick nimmt. Auch die (auf absolut hohem Niveau erfolgten) relativen Emissionsreduktionen seit 1990 beruhen wesentlich auf statistischen Schönungen,

8 Die Frage, mit welchen Strategien solche Reduktionen zu erreichen wären, was also technische Konsistenz- und Effizienzlösungen leisten können, was Suffizienz leistet und was negative Emissionstechnologien leisten könnten (mit welchen Vor- oder Nachteilen), ist kein näherer Gegenstand der vorliegenden Untersuchung; näher z.B. Ekardt 2016a; Ekardt 2018a und kurz oben zu den negativen Emissionen sowie unten zu den Grenzen von Technik. die sich in Wirklichkeit in - wenn man die Konsumemissionen pro Kopf ermittelt - gestiegene Emissionen verkehren (zur Vermeidung von Wiederholungen sei erneut verwiesen auf die nähere Darlegung m.w.N. bei Ekardt 2016a: $\$ 1$ B. III.; Ekardt 2016b; Becker/Richter 2015). Wie die EU gemeinsam mit anderen Vorreiterstaaten durch eine gründliche Revision des ohnehin stark reformbedürftigen Emissionshandels mit sodann strengeren und sukzessive anziehenden Caps sowie einer Erfassung sämtlicher fossiler Brennstoffe auf der ersten Handelsstufe statt nur in einzelnen Sektoren (kombiniert mit Border Adjustments) diese Situation grundlegend verändern könnte, wird hier zur Vermeidung von Wiederholungen nicht erneut dargelegt (siehe m.w.N. Ekardt 2016a: \ 6 E.; Hennig 2017; Bosnjak 2015; teilweise auch von Bredow 2013; allgemein $\mathrm{zu}$ ökonomischen Instrumenten auch Ekardt 2016b).

Eine naheliegende Erklärung, warum (jenseits komplexer verhaltenswissenschaftlicher Erklärungen; dazu Ekardt 2016a: $\S 2$; Ekardt 2017) die Reichweite des Art. 2 Abs. 1 PA konsequent verdrängt wird, dürfte die Folgende sein, die andernorts näher ausgeführt wurde und hier deshalb lediglich in Erinnerung gerufen sei (dazu und zum gesamten vorliegenden Abschnitt Jackson 2011; Paech 2012; Ekardt 2016a: $\$ 1$ B. V.; Ekardt 2016b; Hennig 2017; Stengel 2011; Scheidler 2015). Die völkerrechtlich verbindlichen Temperaturgrenzen aus Art. 2 Abs. 1 PA könnten, wenn sie denn noch umgesetzt würden, sukzessive in eine Welt ohne Wirtschaftswachstum führen. Klimaschutz und Wachstum sind zwar nach verbreiteter (und wohl zutreffender) Einschätzung kompatibel, solange man allein bessere Technik wie erneuerbare Energien und Energieeffizienz zur Ersetzung der fossilen Brennstoffe bei Strom, Wärme, Treibstoff usw. avisiert, denn neue Technik als solche kann man kurz gesagt verkaufen und damit Wachstum schaffen. Trotz aller Unvorhersehbarkeit der (auch technischen und verstärkt dienstleistungswirtschaftlichen und damit emissionsärmeren) Zukunft erreicht man allein mit Technik jedoch die genannten Ziele aus einer Reihe von Gründen wohl eher nicht. Die zwei wichtigsten sind: Die Herausforderung ist angesichts der knappen Zeit bis Ende der 2020er- bzw. Ende der 203oer-Jahre schlicht zu groß; für manche Emissionen (etwa in der Landwirtschaft) fehlen auch technische Optionen. Die mitunter monothematische Ausrichtung der aktuellen Umwelt(rechts) debatte auf das Klimathema verdeckt zudem: Andere Umweltprobleme wie die Degradation von Böden und Ökosystemen gefährden den Menschen ebenfalls auf 
Dauer existenziell und müssen gleichzeitig angegangen werden. Die umweltfachlich naheliegende Lösung, den Nutzungsdruck auf Natur, Böden etc. zu reduzieren, lässt sich noch weniger rein technisch ausführen als ein wirksamer Klimaschutz. Neben grüner Technik gehört zum Klimaschutz unter dem Paris-Abkommen damit wohl neben technischen Maßnahmen auch (!) ein genügsamerer Lebensstil. Weniger Urlaubsflüge, weniger tierische Nahrungsmittel (deren Produktion bisher rund vier Fünftel der weltweiten Agrarflächen beansprucht), weniger Autos u.a.m. implizieren indes ein Ende der Wachstumsgesellschaft. Umgekehrt jedoch hängen am Wirtschaftswachstum bislang zentrale gesellschaftliche Institutionen wie der Arbeitsmarkt, das Renten- und das Bankensystem (näher: Ekardt 2016a: $₫ 1$ B V.).

Insofern gibt das rechtsverbindliche Pariser Langfristziel den Staaten nicht nur sukzessive, aber drastische Aufbesserungen ihrer nationalen Reduktionszusagen auf. Sie erzwingt auch eine umfassende Diskussion über die Zukunft menschlichen Wirtschaftens und Zusammenlebens, der nicht weiter ausgewichen werden kann. Wenn aktuell seit der Bonner Klimakonferenz vom November 2017 angestrebt wird, diese nötigen Nachbesserungen in verkleinerten Gesprächsrunden - nach dem der Fidschi-Sprache entlehnten Talanoa-Prinzip - auf den Weg zu bringen, so bleibt zu hoffen, dass die mit alledem umrissene Problemdimension adäquat aufgegriffen wird.

\section{Literatur}

Anderson, K./Broderick, J. (2017): Natural Gas and Climate Change. Online: http://www.foeeurope.org/sites/default/ files/extractive_industries/2017/natural_gas_and_climate_change_anderson_broderick_october2017.pdf [12.12.2017].

Arndt, B. (2009): Das Vorsorgeprinzip in der Europäischen Union. Tübingen: Mohr Siebeck.

Bauriedl, S. (Hg.) (2016): Wörterbuch Klimadebatte. Bielefeld: Transcript.

Becker, B./Richter, C. (2015): Klimaschutz in Deutschland - Realität oder Rhetorik? Momentum Quarterly, 4 (1), 3-20.

Berger, J./Günther, D./Hain, B. (2016): Das Übereinkommen von Paris - ein wichtiger Wegweiser für eine lebenswerte Zukunft und einen Politikwandel in Deutschland. Zeitschrift für Umweltpolitik und Umweltrecht, Sonderheft, 4-12.
Bodirsky, B./Rolinski, S./Biewald, A./Weindl, I./Popp, A./ Lotze-Campen, H. (2015): Global Food Demand Scenarios for the $21^{\text {st }}$ Century. PLOS ONE, 10 (11). DOI:10.1371

Bosnjak, N. (2015): Ein Emissionshandelssystem der ersten Handelsstufe. Rechtliche, politische und ökonomische Aspekte eines Gesetzgebungsvorschlags. Marburg: Metropolis.

von Bredow, H. (2013): Energieeffizienz als Rechts- und Steuerungsproblem. Unter besonderer Berücksichtigung der erneuerbaren Energien. Marburg: Metropolis.

Buhofer, S. (2017): Der Klimawandel und die internationale Klimapolitik in Zahlen. München: Oekom.

Calliess, C. (2001): Rechtsstaat und Umweltstaat. Tübingen: Mohr Siebeck.

Canadell, P./Le Quéré, C./Peters, G. (2017): We can still keep global warming below $2^{\circ} \mathrm{C}$ - but the hard work is about to start. The Conversation. Online: https://theconversation. com/we-can-still-keep-global-warming-below-2-butthe-hard-work-is-about-to-start-72075 [02.08.2017].

Drouet, L/ Emmerling, J. (2016): Climate policy under socioeconomic scenario uncertainty. Environmental Modelling \& Software, 79, 334-342.

Ekardt, F. (2018): Ökonomische Bewertung - Kosten-NutzenAnalyse - ökonomische Ethik. Eine Kritik unter besonderer Berücksichtigung von Nachhaltigkeit und Klimaschutz. Marburg: Metropolis, i.E.

Ekardt, F./Zorn, A. (2018): Ozeanversauerung, Meeresumweltrecht, Klimavölkerrecht und die Menschenrechte, Jahrbuch des Umwelt- und Technikrechts, i.E.

Ekardt, F. (2017): Rezension zu Dieckhoff, Modellierte Zukunft und Dieckhoff/Leuschner, Die Energiewende und ihre Modelle, Zeitschrift für Umweltpolitik und Umweltrecht, 3, 282-285.

Ekardt, F./Wieding, J. (2016): Rechtlicher Aussagegehalt des Paris-Abkommen - eine Analyse der einzelnen Artikel. Zeitschrift für Umweltpolitik und Umweltrecht, Sonderheft, 36-57.

Ekardt, F. (2016a): Theorie der Nachhaltigkeit. Ethische, rechtliche, politische und transformative Zugänge - am Beispiel von Klimawandel, Ressourcenknappheit und Welthandel. 3. Auflage $(=2$. Aufl. der Neuausgabe) Baden-Baden: Nomos.

Ekardt, F. (2016b): Zur Verteidigung ökonomischer Politikinstrumente gegen ihre Freunde und Kritiker: Analysen des Hauptinstruments der Transformation zur Nachhaltigkeit. Momentum Quarterly, 5 (4), 224-242.

Ekardt, F./Wieding, J./Henkel, M. (2015): Climate Justice 2015 - BUNDposition. Berlin. Online: https://www.bund.net/ service/publikationen/detail/publication/climate-justice-2015/ [02.10.2017].

Figueres, C. u.a. (2017): Three years to safeguard our climate. Nature, 546 (7660). Online: https://www.nature. $\mathrm{com} /$ news/three-years-to-safeguard-our-climate-1.22201 [21.10.2017].

Friedrich, T./Timmermann, A./Tigchelaar, M./Timm, O./ Ganopolski, A. (2016): Nonlinear climate sensitivity and 
its implications for future greenhouse warming. Science Advances, 2 (11). Online: http://advances.sciencemag. org/content/2/11/e1501923.full [02.08.2017].

Frieler, K./Meinshausen, M./Golly, A./Mengel, M./Lebek, K./ Donner, S./Hoegh-Guldberg, O. (2013): Limiting global warming to $2^{\circ} \mathrm{C}$ is unlikely to save most coral reefs. $\mathrm{Na}$ ture Climate Change, 3, 165-170.

Hennig, B. (2017): Nachhaltige Landnutzung und Bioenergie. Ambivalenzen, Governance, Rechtsfragen. Marburg: Metropolis.

Höhne, N./Kuramochi, T./Sterl, S./Röschel, L. (2016): Was bedeutet das Pariser Abkommen für den Klimaschutz in Deutschland? New Climate Institute/ Greenpeace, Berlin.

Hoffmann, U. (2015): Can Green Growth really Work and what are the True (Socio-)Economics of Climate Change? Online: http://unctad.org/en/PublicationsLibrary/osgdp2015d4_en.pdf [02.08.2017].

IPCC (2013): Climate Change 2013. The Physical Science Basis. Contribution of Working Group I to the Fifth Assessment Report of the Intergovernmental Panel on Climate Change. Cambridge, New York: Cambridge University Press.

IPCC (2014a): Climate Change 2014. Synthesis Report. Contribution of Working Groups I, II and III to the Fifth Assessment Report of the Intergovernmental Panel on Climate Change. Geneva.

IPCC (2014b): Climate Change 2014. Mitigation of Climate Change. Working Group III Contribution to the Fifth Assessment Report of the Intergovernmental Panel on Climate Change. Cambridge, New York: Cambridge University Press.

Jackson, T. (2011): Wohlstand ohne Wachstum. München: Oekom.

Knox, J. (2013): Report of the Independent Expert on the issue of human rights obligations relating to the enjoyment of a safe, clean, healthy and sustainable environment, UN Doc. A/HRC/25/53 vom 30.12.2013.

Knutti, R./Rogelj, J./Sedláček, J./Fischer, E. (2016): A scientific critique of the two-degree climate change target. Nature Geoscience, 9, 13-18. Online: http://www.nature.com/ ngeo/journal/v9/n1/full/ngeo2595.html [02.08.2017].

Koch, T. (200o): Der Grundrechtsschutz des Drittbetroffenen. Tübingen: Mohr Siebeck.

Le Quéré, C. u.a. (2016): Global Carbon Budget 2016. Earth Syst. Sci. Data, 8, 605-649. Online: https://doi. org/10.5194/essd-8-605-2016 [24.09.2017].

Mauritsen, T./ Pincus, R. (2017): Committed warming inferred from observations. Nature Climate Change, 7, 652655. Online: http://www.nature.com/nclimate/journal/ $\mathrm{v} / \mathrm{ng} / \mathrm{full} / \mathrm{nclimate} 3357 . \mathrm{html}$ [24.09.2017].

Maurmann, D. (2008): Rechtsgrundsätze im Völkerrecht - am Beispiel des Vorsorgeprinzips. Baden-Baden: Nomos.

Meßerschmidt, K. (2000): Gesetzgebungsermessen. Berlin: Verlag Arno Spitz.

Monien, J. (2014): Prinzipien als Wegbereiter eines globalen Umweltrechts? Das Nachhaltigkeits-, Vorsorge- und Ver- ursacherprinzip im Mehrebenensystem. Baden-Baden: Nomos.

Moreno, C./Speich Chassé, D./Fuhr, L. (2015): Carbon Metrics. Global Abstractions and Ecological Epistemicide. Berlin: Heinrich Böll Stiftung. Online: https://www.boell.de/de/node/287891 [21.01.2018].

Millar, R. u.a. (2017): Emission budgets and pathways consistent with limiting warming to $1.5^{\circ} \mathrm{C}$. Nature Geoscience, 10, 741-747. DOI: 10.1038/NGEO3031.

Peters, G. (2017): How much carbon dioxide can we emit? Cicero. Online: http://www.cicero.uio.no/no/posts/klima/ how-much-carbon-dioxide-can-we-emit [02.08.2017].

Paech, N. (2012): Befreiung vom Überfluss. München: Oekom.

Paqué, K.-H. (2010): Wachstum! Die Zukunft des globalen Kapitalismus. München: Hanser.

Peters, G./Andrew, R. M./Karstensen, J. (2016): Global environmental footprints: A guide to estimating, interpreting and using consumption-based accounts of resource use and environmental impacts. Nordic Council of Ministers, Copenhagen. Online: http://dx.doi.org/10.6027/tn2016532 [24.09.2017].

Peters, G. (2017): How much carbon dioxide can wie emit? CICERO, 16.03.2017. Online: http://www.cicero.uio.no/ no/posts/klima/how-much-carbon-dioxide-can-weemit [21.10.2017].

Piketty, T. (2015): Das Kapital im 21. Jahrhundert. Bonn: BpB.

Raftery, A. E./Zimmer, A./Frierson, D.M.W./Startz, R./Liu, Peiran (2017): Less than $2^{\circ} \mathrm{C}$ warming by 2100 unlikely. Nature Climate Change, 7, 637-641. Online: http://www. nature.com/nclimate/journal/v7/ng/full/nclimate3352. html [24.09.2017].

Rahmstorf, S. (2017): Die Koalitionsgespräche und das deutsche Emissionsbudget. Spektrum Scilogs, 17.10.2017. Online: https://scilogs.spektrum.de/klimalounge/die-koalitionsgespraeche-und-das-deutsche-emissionsbudget/ [21.10.2017].

Rahmstorf, S./Levermann, A. (2017): 2020. The Climate Turning Point. Online: http://www.mission2020.global/2020\%20The\%20Climate\%2oTurning\%2oPoint.pdf (12.12.2017).

Rajamani, L. (2010): The Increasing Currency and Relevance of Rights-based Perspectives in the International Negotiations on Climate Change. Journal of Environmental Law, 22 (3), 391-429.

Rogelj, J./Schaeffer, M./Friedlingstein, P./Gillett, N. P./van Vuuren, D. P./Riahi, K./Allen, M./Knutti, R. (2017): Differences between carbon budget estimates unravelled. Nature Climate Change, 6, 245-252.

Rogelj, J./ den Elzen, M./ Höhne, N./Fransen, T./Fekete, H./ Winkler, H/Schaeffer, R./Sha, F./Riahi, K./Meinshausen, M. (2016): Paris agreement climate proposals need a boost to keep global warming well below 2 degrees Celsius. Nature, 534 (7609), 631-639.

Rose, S./Richels, R./Blanford, G./Rutherford, T. (2017): The Paris Agreement and next steps in limiting global warming. Climatic Change, 142 (1-2), 255-270. 
Scheidler, F. (2015): Das Ende der Megamaschine. Geschichte einer scheiternden Zivilisation. Wien: Promedia.

Schellnhuber, H.-J. (2015): Selbstverbrennung. Die fatale Dreiecksbeziehung zwischen Klima, Mensch und Kohlenstoff. München: C. Bertelsmann Verlag.

Schellnhuber, H.-J./Rahmstorf, S./Winkelmann, R. (2016): Why the right climate target was agreed in Paris. Nature Climate Change, 6, 649-653.

Schmidt, G. (2007): Climate models produce projections, not probabilities. The uncertainty in climate modeling. Bulletin of the Atomic Scientists. Online: http://thebulletin. org/uncertainty-climate-modeling [03.08.2017].

Schmidt-Radefeldt, R. (2000): Ökologische Menschenrechte. Baden-Baden: Nomos.

Schurer, A./ Mann, M./Hawkins, E./Tett, S./Hegerl, G. (2017): Importance of the pre-industrial baseline for likelihood of exceeding Paris goals. Nature Climate Change, 7, 563-567. Online: http://www.nature.com/ nclimate/journal/vaop/ncurrent/full/nclimate3345. html?foxtrotcallback=true\#ref2 [01.08.2017].

Skillington, T. (2012): Climate Change and the Human Rights Challenge. Extending Justice beyond the Borders of the Nation State. International Journal of Human Rights, 16 (8), 1196-1212.

Smith, P./Davis, S.J./Creutzig, F./Fuss, S./Rogelj, J./McCollum, D./Krey, V./Grubler, A. et al. (2016): Biophysical and economic limits to negative $\mathrm{CO}_{2}$ emissions. Nature Climate Change, 6 (1), 42-50. DOI:10.1038/nclimate2870 [24.09.2017].

Steininger, K./Meyer, L. (2017): Das Treibhausgas-Budget für Österreich. Online: https://wegcwww.uni-graz.at/publ/ wegcreports/2017/WCV-WissBer-Nr72-LMeyerKSteininger-Okt2017.pdf [12.12.2017].

Stengel, O. (2011): Suffizienz. Die Konsumgesellschaft in der ökologischen Krise. München: Oekom.

Storelvmo, T./Tan, I./Zelinka, M. (2016): Observational constraints on mixed-phase clouds imply higher climate sensitivity. Science, 352 (6282), 224-227.

Susnjar, D. (2010): Proportionality, Fundamental Rights and Balance of Powers. Brill: Leiden.

Tollefson, J. (2015): Is the $2{ }^{\circ} \mathrm{C}$ world a fantasy? Nature, 527 (7579). Online: http://www.nature.com/news/is-the-2-cworld-a-fantasy-1.18868 [03.08.2017].

UNEP (2016): The Emissions Gap Report 2016. United Nations Environment Programme (UNEP), Nairobi.

UNEP (2017): The Emissions Gap Report 2017. United Nations Environment Programme (UNEP), Nairobi. Online: https://wedocs.unep.org/bitstream/handle/20.500.11822/22070/EGR_2017.pdf [12.12.2017].

Unnerstall, H. (1999): Rechte zukünftiger Generationen. Würzburg: Königshausen \& Neumann.

Verheyen, Roda (2005): Climate Change Damage and International Law. Berlin u.a.: Springer.

Wiebe, K. u.a. (2015): Climate change impacts on agriculture in 2050 under a range of plausible socioeconomic and emissions scenarios. Environmental Research Letters, 10 (8). DOI:10.1088/1748-9326/10/8/085010. 